\title{
Intraoral Botulinum Toxin Injection in Mental Foramen Site in the Treatment of Trigeminal Neuralgia
}

\author{
Alireza Nvabazam ${ }^{1}$, Reza Judi² \\ ${ }^{1,2}$ Department of Maxillofacial Surgery, Shahid Saduqi University of Medical Sciences, Yazd, Iran.
}

\section{ABSTRACT}

\section{BACKGROUND}

Botulinum toxin type-A (BTX-A), has been used successfully in trigeminal neuralgia treatment. In this research, a novel method is proposed to assess the effectiveness of the BTX-A injection to the mental nerve. The present study aimed to evaluate BTX-A injection effectiveness in the mental nerve of trigeminal neuralgia (TN) patients who had a trigger zone in the mental nerve distribution.

\section{METHODS}

In this quasi-experimental, pre-and post-test design without control group research, 50 units of BTX-A was injected into the mental foramen site in 20 patients. Pain frequency and Visual Analog Scale (VAS) was evaluated prior to the treatment and in the first week, the second month, the sixth month, and the first year after treatment. Responders were those patients with a decrease of 50 percent in the score of mean pain in the second and sixth month.

\section{RESULTS}

The study included 20 patients. Pain intensity and attack frequency was decreased significantly by BTX-A. Ninety-five percent of patients reacted to therapy in the first year. After an average period of $165 \pm 31.6$ (44 - 235) days, 50 percent of patients encountered recurrence and, for a second time, received treatment.

\section{CONCLUSIONS}

Injection appears to be a very successful technique in the mental foramen site; the attack frequency and pain severity are reduced after each injection. Based on the present research, intraoral injection of BTX-A to the mental foramen site is an efficient and safe treatment. In TN and possibly other similar disorders, BTX-A can be a beneficial treatment technique. A fast and long-term advantage is provided by BTXA, without systemic effects.

\section{KEY WORDS}

Botulinum Toxin, Trigeminal Neuralgia Treatment
Corresponding Author:

Reza Judi,

Department of Maxillofacial Surgery,

Shahid Saduqi University of Medical

Sciences, Yazd, Iran.

E-mail: judi.omfs@gmail.com

DOI: $10.14260 /$ jemds/2021/49

How to Cite This Article:

Nvabazam A, Judi R. Intraoral botulinum toxin injection in mental foramen site in the treatment of trigeminal neuralgia. J Evolution Med Dent Sci 2021;10(04):227230, DOI: $10.14260 / \mathrm{jemds} / 2021 / 49$

Submission 01-09-2020,

Peer Review 27-11-2020,

Acceptance 04-12-2020,

Published 25-01-2021.

Copyright (c) 2021 Alireza Nvabazam et al. This is an open access article distributed under Creative Commons Attribution License [Attribution 4.0 International (CC BY 4.0)] 


\section{BACKGROUND}

According to the International Association for the Study of Pain (IASP), trigeminal neuralgia is a unilateral facial pain syndrome defined by sharp, brief, paroxysmal lancinating pains recurred and limited to the distribution of trigeminal nerve divisions. ${ }^{1}$ Its prevalence is around 1 in $25000 .{ }^{2}$ Idiopathic cases, besides ones with the fifth cranial nerve's vascular compression are considered as classical TN (CTN); symptomatic TN (STN) is observed in secondary cases of tumours, multiple sclerosis, and structural abnormalities in the skull base. ${ }^{3}$ At first, pharmacological agents typically are used for treating TN patients. ${ }^{4}$ In almost $80 \%$ of patients, the medicine may help to handle the pain.

Carbamazepine is the first line of treatment that relieves most of the symptoms detected. In most TN patients, other medicines, such as phenytoin, baclofen, oxcarbazepine, lamotrigine, sodium valproate, and gabapentin, effectively decrease the symptoms as well. Medicines in the TN treatment have some side effects, including lethargy, dizziness, fatigue, vomiting, nausea, reversible thrombocytopenia, occasional granulocyte reduction, or hepatitis associated aplastic anaemia. With inadequate effects or undesirable side effects of pharmacological therapy, surgery is a choice. Since TN involves percutaneous radiofrequency thermal rhizotomy, neurectomy of the trigeminal nerve branches outside the skull, percutaneous ablation using heat to generate the trigeminal nerve or trigeminal ganglion lesions, glycerol injection into the trigeminal cistern, percutaneous retrogasserian glycerol rhizotomy, physical compression, cryotherapy, alcohol injections, trigeminal ganglion balloon micro compression, botulinum toxin injection, and gamma knife radiosurgery (GKRS), a number of surgical methods are applied for relieving the pain. Many surgical processes can result in certain complications, ${ }^{5}$ including facial paraesthesia, hearing loss, hypoesthesia, keratitis, masseter weakness and paralysis, transient paralysis of cranial nerves III and VI, such as dysesthesia, diminished corneal reflex, and anaesthesia Dolorosa, or immediate complete vision loss in one eye after trigeminal radiofrequency rhizotomy because of acute traumatic optic neuropathy. ${ }^{6}$

TN may be relieved by the injection of BTX-A in trigger zones. ${ }^{7-10}$ In TN treatment, hematoma / oedema and facial asymmetry, including the commissure distortion, muscle relaxation, and the eyelid ptosis at the injection site, are the frequent side effects caused by botulinum toxin. All such side effects have been mild and vanished automatically with no more treatment. There have been no systemic side effects. ${ }^{11}$, BTX-A has been administered intradermally and subcutaneously in all the research. The present study aimed to evaluate BTX-A injection effectiveness in the mental nerve of TN patients who had a trigger zone in the mental nerve distribution.

\section{METHODS}

In this quasi-experimental, pre-and post-test design without control group research, according to the Börü et al study and the calculation of standard deviation of VAS before and after the intervention were 0.6 and 2.4, respectively, and the minimum difference between the means of 1.5 and a $95 \%$ confidence level and the power of $80 \%, 20$ samples were examined. 12

Before the treatment, the objective, process, and safety aspects of this study were clarified to all patients. From April 2017 to September 2018, all patients referred to the Maxillofacial Surgery Polyclinic and diagnosed with classical TN were included in the study. In order to avoid the existence of structural pathology, each patient went through magnetic resonance imaging. They were diagnosed with classical TN, based on the International Classification of Headache Disorders (ICHD-2), ${ }^{13}$ Further, patients' demographic characteristics, trigger factors, involvement side, disease duration, and medicines were recorded before treatment. Medicines, including gabapentin, carbamazepine, amitriptyline, and pregabalin, were used by most patients at the baseline. In the study period, these medicines were stopped, and no new ones were taken. In this study, the inclusion criteria were the failure in the present treatment, baseline pain intensity of $\geq 4$, and attack frequency of $\geq 4$ per day.

Further, exclusion criteria were any systemic illness or any agent use potential to interfere with BTX-A. Moreover, all patients referred to the Maxillofacial Surgery Polyclinic and not diagnosed with classical TN were excluded, finally pregnant women or those with a plan of pregnancy during the treatment period were excluded. Enrolment continued until twenty patients of classical TN were found $.0 .5 \mathrm{mg}$ of human albumin, $0.9 \mathrm{mg}$ of sodium chloride, and 300 Units (U) of Clostridium botulinum type A neurotoxin complex form the content of each Dysport vial (botulinum toxin A); this content first was diluted in $3 \mathrm{~mL}$ saline solution (0.9\%). Then, $50 \mathrm{U}$ $(0.5 \mathrm{~mL})$ was injected at every site. To administer toxin in all patients, a 28-gauge insulin needle was applied, and all injections to the patients was done by one professional maxillofacial surgeon.

The patients were seated with heads supported by a headrest. Their mouths were open to some extent, and the needle was pointed to the mandibular bone for the injection to the mental nerve, through the mandibular buccal vestibule between lower premolars at a depth of $5 \mathrm{~mm}$ around the mental foramen; the solution was administered around the mental nerve. Throughout the process, the echo graphic or radioscopic guide was not applied. Even though most patients have been followed-up up until now, the study consisted of the patients' one-year follow-up after treating with BTX-A. Before starting the study, baseline examinations were carried out. In the first week, the second month, the sixth month, and one year, the patients were examined.

Then, the details of adverse effects and pain severity were requested. On the basis of the Visual Analog Scale, pain severity related to chewing, tooth brushing, and cold weather was documented. Patients were requested to contact the hospital when they experienced the pain in this period. Patients were examined once more when the pain recurred, and if necessary, injections were given again. The pain severity was assessed using the VAS (the 11-point visual analog score). From baseline to endpoint, $\geq 50 \%$ decrease in mean pain score was considered a reaction to treatment. At each visit, the negative effects were documented.

Percentages and distributions of frequency were computed. We used the Wilcoxon signed-rank test to compare 
the VAS and the frequency before and after the intervention. At the level of $\mathrm{P}<.05$, results were statistically significant. The SPSS software version 21 was used to carry out tests.

The research ethics committee of the Shahid Saduqi University of Medical Science reviewed and approved the study protocol and procedures with No IR.IUMS.REC.1397.478.

\section{RESULTS}

The study included 20 patients with the age range of $37-77$ years (the mean age: $54.8 \pm 4.5$ ). Of them, ten were females ( 50 $\%)$ and ten were males (50\%). The mean disease period was $5.3 \pm 2.8$ years. Before the intervention, the mean score of baseline pain (VAS) was $9.8 \pm 0.5(\mathrm{p}=.06)$. At the first week, the second month, the sixth month, and the first year, this score was $3.8 \pm 1.2,2.2 \pm 0.8,1.9 \pm 0.9$, and $1.2 \pm 0.8$, respectively. Significant differences was observed between the score of baseline VAS and those of the first week, the second month, the sixth month, and the first year $(\mathrm{P}=.001, \mathrm{P}=.001, \mathrm{P}$ $=.001$, and $\mathrm{P}=.001)$. The number of attacks per day was used to calculate attack frequency.

Before the intervention, the mean baseline attack frequency was $311.6 \pm 81.5$; further, it was $83.5 \pm 28.2,64.4 \pm$ $23.5,42.1 \pm 19.2$, and $24.3 \pm 10.7$ at the first week, the second month, the sixth month, and the first year, respectively. A statistically significant difference was observed between the frequency at baseline and that in the first week, the second month, and the sixth month $(\mathrm{P}=.001, \mathrm{P}=.001, \mathrm{P}=.001$, and $\mathrm{P}$ $=.001$ ) (Table 1). Reaction to the treatment was considered to be $\mathrm{a} \geq 50 \%$ reduction in pain score from baseline to endpoint. Patients response to the treatment, on the basis of this criteria, was $15(75 \%), 17$ (85\%), 18 (90\%), and 19 (95\%) at the first week, the second month, the sixth month, and the first year, respectively. At the end of the first year, 12 (60\%) patients were pain-free.

After the mean period of $165 \pm 31.6$ (44 - 235) days, 10 (50 $\%)$ patients encountered recurrence; thus, for a second time, they were treated. 1 patient did encounter no change in pain severity and frequency, although he received a third injection throughout the one-year period. Negative effects: 1 patient encountered short-term inferior labial weakness on the side of injection; this effect vanished in 2 months.

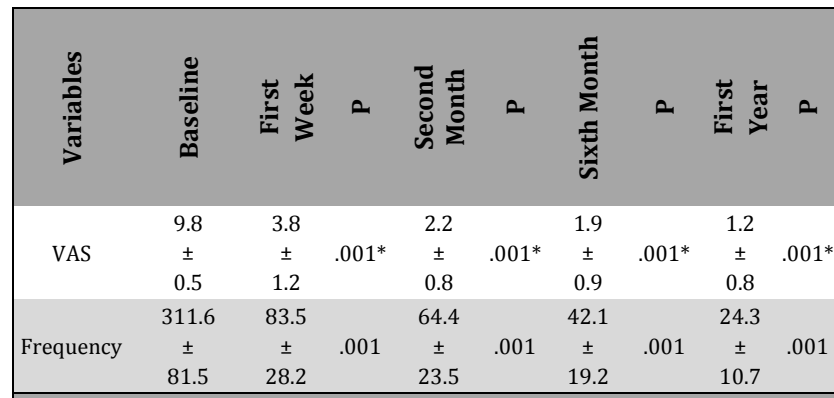

Table 1. Visual Analogue Score (VAS) and Frequency Changes *the Wilcoxon signed-rank test

\section{DISCUSSION}

Based on the results, at each follow-up, attack frequency and pain intensity in patients who received BTX-A improved clinically and statistically. A recurrence was not seen in the sixth month in about half of the patients; however, it developed in the remaining half. The patients' follow-up is continuing. There is also an attempt to publish long-term research results. The attack frequency and pain severity were reduced increasingly. In the present work, a similar technique to other authors was not used. In the first year, pain severity was reduced by about $95 \%$, according to this technique. In the last decade, BTX-A has been used in TN; in nearly all research, BTX-A injected intracutaneously, subcutaneously, and into the trigger zone in the painful area of the face.9,14-16 In the present research, $50 \mathrm{U}$ of BTX-A was injected in the mental foramen site. According to Wu et al., BTX-A was safe and efficient in the TN treatment; it was injected by them in the painful area intradermally and submucosally; their reaction rate was as high as $70 \%{ }^{14} 86 \mathrm{TN}$ patients were treated by Jian-Huo Xia et al.; they also injected it intracutaneously in the painful area of the face, reaching the effectiveness rate at week 8 by $80 \%$. Based on other research, BTX-A injection in the painful area of the face relieves pain significantly in TN. ${ }^{15,16}$ According to a recent study by Batifol et al., a low dose of injection is needed into the trigger zone.

Also, they showed a high response rate to BTX-A. ${ }^{17}$ In a single-blinded randomised placebo-controlled research, Sheta et al. reported a significant pain decrease in the BTX-A group as compared with the placebo group. They further showed a significant rise in quality of life and a reduction in the number of acute medicine. ${ }^{18}$ In a double-blinded randomised placebocontrolled trial, Zhang et al. used $25 \mathrm{U}$ for one group and $75 \mathrm{U}$ for the other; then, they compared the results with a placebo group. Based on the results, in BTXA groups, compared to the placebo group, the reaction rates were higher significantly; however, no statistically significant difference was observed between $25 \mathrm{U}$ and $75 \mathrm{U}$ groups. Just 4 randomised controlled investigations have been conducted up to 2016, according to systematic review. One hundred seventy-six patients with TN were in total treated. Based on their results, BTX-A is a highly efficient and useful substance compared to the placebo in the TN treatment. Their injection method was intradermal and / or subcutaneous. ${ }^{19}$ The injection in the present research method was administered nearby mental nerve. A substantial success was observed in this technique. Compared to other methods, it was slightly more effective. Further, in this work, no substantial safety concerns were observed.

\section{CONCLUSIONS}

Based on the present research, the intraoral injection of BTXA to the mental foramen site is an efficient and safe treatment. In TN and possibly other similar disorders, BTX-A can be a beneficial treatment technique. It is fast acting and provides long-term relief without systemic effects. Further double-blind trials with the right number of patients would confirm our results and investigate if higher doses have a longer-term impact and to optimise injection sites. Also, more studies can help to explain optimal schedules and methods for injection. 
Data sharing statement provided by the authors is available with the full text of this article at jemds.com.

Financial or other competing interests: None.

Disclosure forms provided by the authors are available with the full text of this article at jemds.com.

We would like to thank all patients involved in this research.

\section{REFERENCES}

[1] Ngeow WC, Nair R. Injection of botulinum toxin type A (BOTOX) into trigger zone of trigeminal neuralgia as a means to control pain. Oral Surg Oral Med Oral Pathol Oral Radiol Endod 2010;109(3):e47-50.

[2] Castillo-Alvarez F, de la Barcena IH, Marzo-Sola ME, et al. Botulinum toxin in trigeminal neuralgia. Med Clin (Barc) 2017;148(1):28-32.

[3] Hu Y, Guan X, Fan L, et al. Therapeutic efficacy and safety of botulinum toxin type A in trigeminal neuralgia: a systematic review. J Headache Pain 2013;14(1):72.

[4] Antonaci F, Arceri S, Rakusa M, et al. Pitfals in recognition and management of trigeminal neuralgia. J Headache Pain 2020;21(1):82.

[5] Heinskou TB, Maarbjerg S, Wolfram F, et al. Favourable prognosis of trigeminal neuralgia when enrolled in a multidisciplinary management program-a two-year prospective real-life study. J Headache Pain 2019;20(1):23.

[6] Zhang H, Lian Y, Ma Y, et al. Two doses of botulinum toxin type A for the treatment of trigeminal neuralgia: Observation of therapeutic effect from a randomized, double-blind, placebo-controlled trial. J Headache Pain 2014;15(1):65.

[7] Li S, Lian YJ, Chen Y, et al. Therapeutic effect of botulinum toxin-a in 88 patients with trigeminal neuralgia with 14month follow-up. J Headache Pain 2014;15(1):43.

[8] Fabregat G, De Andrés J, Villanueva-Pérez VL, et al. Subcutaneous and perineural botulinum toxin type A for neuropathic pain: a descriptive review. Clin J Pain 2013;29(11):1006-12.
[9] Prasad S, Galetta S. Trigeminal neuralgia: historical notes and current concepts. Neurologist 2009;15(2):87-94.

[10] Turton M, Malan-Roux P. Trigeminal neuralgia: case report and literature review. Stomatological Dis Sci 2019;3:7.

[11] Piovesan EJ, Teive HG, Kowacs PA, et al. An open study of botulinum - a toxin treatment of trigeminal neuralgia. Neurology 2005;65(8):1306-8.

[12] Börü ÜT, Duman A, Bölük C, et al. Botulinum toxin in the treatment of trigeminal neuralgia: 6-month follow-up. Medicine (Balimore) 2017;96(39):e8133.

[13] Piovesan EJ, da Silva Leite L, Teive HG, et al. Botulinum toxin type A effect as a preemptive treatment in a model of acute trigeminal pain: a pre-clinical double-blind and placebo-controlled study. Arq Neuropsiquiatr 2011;69(1):56-63.

[14] Wu CJ, Lian YJ, Zheng YK, et al. Botulinum toxin type A for the treatment of trigeminal neuralgia: results from a randomized, double-blind, placebo-controlled trial. Cephalalgia 2012;32(6):443-50.

[15] Xia JH, He CH, Zhang HF, et al. Botulinum toxin an in the treatment of trigeminal neuralgia. Int J Neurosci 2016;126(4):348-53

[16] Burmeister J, Holle D, Bock E, et al. Botulinum neurotoxin type $A$ in the treatment of classical trigeminal neuralgia (BoTN): study protocol for a randomized controlled trial. Trials 2015;16:550.

[17] Batifol D, Finiels PJ. Botulinum toxin in trigeminal neuralgia: which is the best way of delivery? J Spine Neurosurg 2016;5:4.

[18] Shehata H, El-Tamawy M, Shalaby N, et al. Botulinum toxin-type A: could it be an effective treatment option in intractable trigeminal neuralgia? J Headache Pain 2013;14(1):92.

[19] Morra M, Elgebaly A, Elmaraezy A, et al. Thrapeutic efficacy and safety of botulinum toxin A therapy in trigeminal neuralgia: a systematic review and metaanalysis of randomized controlled trials. J Headache Pain 2016;17(1):63. 